\title{
A High-Fat, High-Fructose Diet Induces Antioxidant Imbalance and Increases the Risk and Progression of Nonalcoholic Fatty Liver Disease in Mice
}

\author{
Kanokwan Jarukamjorn, ${ }^{1}$ Nattharat Jearapong, ${ }^{1}$ \\ Charinya Pimson, ${ }^{1}$ and Waranya Chatuphonprasert ${ }^{2}$ \\ ${ }^{1}$ Research Group for Pharmaceutical Activities of Natural Products Using Pharmaceutical Biotechnology (PANPB), \\ Faculty of Pharmaceutical Sciences, Khon Kaen University, Khon Kaen 40002, Thailand \\ ${ }^{2}$ Faculty of Medicine, Mahasarakham University, Mahasarakham 44000, Thailand \\ Correspondence should be addressed to Kanokwan Jarukamjorn; kanok_ja@kku.ac.th
}

Received 15 December 2015; Accepted 2 February 2016

Academic Editor: Stephen D. H. Malnick

Copyright ( 2016 Kanokwan Jarukamjorn et al. This is an open access article distributed under the Creative Commons Attribution License, which permits unrestricted use, distribution, and reproduction in any medium, provided the original work is properly cited.

Excessive fat liver is an important manifestation of nonalcoholic fatty liver disease (NAFLD), associated with obesity, insulin resistance, and oxidative stress. In the present study, the effects of a high-fat, high-fructose diet (HFFD) on mRNA levels and activities of the antioxidant enzymes, including superoxide dismutase (SOD), catalase (CAT), and glutathione peroxidase (GPx), were determined in mouse livers and brains. The histomorphology of the livers was examined and the state of nonenzymatic reducing system was evaluated by measuring the glutathione system and the lipid peroxidation. Histopathology of the liver showed that fat accumulation and inflammation depended on the period of the HFFD-consumption. The levels of mRNA and enzymatic activities of SOD, CAT, and GPx were raised, followed by the increases in malondialdehyde levels in livers and brains of the HFFD mice. The oxidized GSSG content was increased while the total GSH and the reduced GSH were decreased, resulting in the increase in the GSH/GSSG ratio in both livers and brains of the HFFD mice. These observations suggested that liver damage and oxidative stress in the significant organs were generated by continuous HFFD-consumption. Imbalance of antioxidant condition induced by long-term HFFD-consumption might increase the risk and progression of NAFLD.

\section{Introduction}

Nonalcoholic fatty liver disease (NAFLD) is a condition in which excessive fat accumulates in the liver of a patient who drinks little or no alcohol. The development of NAFLD is associated with obesity, insulin resistance (diabetes mellitus type 2), and hyperlipidemia [1] and is characterized by the accumulation of fatty acids, especially excess triglycerides, in hepatocytes [2]. NAFLD may also be associated with elevated liver enzyme levels, abnormal liver function, and disruption of the metabolism, secretion, and excretion of lipids [3]. Hepatic steatosis (more than $5.5 \%$ hepatic triglyceride content) is the first stage of NAFLD and is followed by liver injury in the next stage of the disease, called nonalcoholic steatohepatitis (NASH) [4]. Cytokine production is altered in the NASH stage which is further characterized by lobular inflammation, hepatocyte ballooning, and fibrosis [5]. Other factors, such as oxidative injury or oxidative stress, are required to shift from NASH to liver cirrhosis, before finally leading to hepatocellular carcinoma, in severe cases [6]. Oxidative stress and lipid peroxidation are crucial pathophysiological mechanisms in NAFLD [7]. Oxidative stress can be detected by measuring the activity of antioxidative enzymes, for example, superoxide dismutase (SOD), catalase (CAT), and glutathione peroxidase (GPx), as well as the level of malondialdehyde (MDA), particularly in the liver. Increases in SOD and GPx activity and SOD, GPx, and CAT mRNA expression in the livers of rabbits fed a high-fat diet have indicated a significant role for the antioxidant system in NAFLD [8].

The increasing prevalence of obesity, metabolic syndrome, and NAFLD has been connected to excess caloric intake, such as the excessive consumption of processed food 
TABLE 1: Forward and reverse primers for mouse CAT, CuZn-SOD, Mn-SOD, GPx, and GAPDH.

\begin{tabular}{|c|c|c|c|}
\hline Gene & Forward primer & Reverse primer & References \\
\hline CAT & 5'-GCA GAT ACC TGT GAA CTG TC-3' $^{\prime}$ & 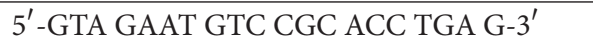 & [17] \\
\hline $\mathrm{CuZn-SOD}$ & $5^{\prime}$-AAG GCC GTG TGC GTG CTG AA-3' & $5^{\prime}$-CAG GTC TCC AAC ATG CCT CT- $3^{\prime}$ & {$[18]$} \\
\hline$M n-S O D$ & $5^{\prime}$-GCA CAT TAA CGC GCA GAT CA-3' & $5^{\prime}$-AGC CTC CAG CAA CTC TCC TT- $3^{\prime}$ & [18] \\
\hline$G P x$ & $5^{\prime}$-CCT CAA GTA CGT CCG ACC TG- $3^{\prime}$ & $5^{\prime}$-CAA TGT CGT TGC GGC ACA CC- $3^{\prime}$ & [18] \\
\hline GAPDH & $5^{\prime}$-TCC ACT CAC GGC AAA TTC AAC G-3' & $5^{\prime}$-TAG ACT CCA CGA CAT ACT CAG C-3' & [19] \\
\hline
\end{tabular}

with high fructose levels [9]. High fructose consumption has been implicated in the progression and severity of NAFLD by promoting de novo lipogenesis and increasing insulin resistance, oxidative stress, inflammation, and fibrosis $[10,11]$; however the mechanism responsible is still unknown [12].

In the current study, we included fructose and transfats in a high-energy diet to induce experimental NAFLD in mice $[10,13]$. Here, we describe the effect of this high-fat, high-fructose diet (HFFD) on the expression and activity of the SOD, CAT, and GPx antioxidant enzymes and the overall oxidative state in terms of total glutathione, reduced glutathione (GSH), and oxidized glutathione (GSSG). This study aims to elucidate the relationship between high-fat, high-fructose consumption, the oxidant-antioxidant balance, and the risk and progression of NAFLD.

\section{Materials and Methods}

2.1. Chemicals. Fructose syrup (55\%) was purchased from Chao Khun Agro Products Co., Ltd. (Bangkok, Thailand). Bovine serum albumin (BSA), 5,5-dithiobis-(2-nitrobenzoic acid) (DTNB), glutathione reductase, oxidized glutathione (GSSG), reduced glutathione (GSH), nitrotetrazolium blue chloride (NBT), reduced $\beta$-nicotinamide adenine dinucleotide phosphate (NADPH), standard malondialdehyde (MDA), standard superoxide dismutase (SOD) from bovine erythrocytes, 4-vinylpyridine (4-VP), and xanthine oxidase were from Sigma Aldrich (St. Louis, MO, USA). Hydrogen peroxide $\left(\mathrm{H}_{2} \mathrm{O}_{2}\right)$ was obtained from Fisher Scientific (Leicestershire, UK). 2-Thiobarbituric acid (TBA) was supplied by Fluka Chemika Co. (Steinheim, Switzerland). Trizol ${ }^{\circledR}$ was supplied by Invitrogen ${ }^{\circ}$ (Carlsbad, CA, USA). ReverTraAce ${ }^{\circ}$ and Taq DNA polymerase were purchased from Toyobo Co., Ltd. (Osaka, Japan) and Vivantis Technologies Sdn. Bhd. (Selangor Darul Ehsan, Malaysia), respectively. The random primers and RNase inhibitor were products of Takara Bio Inc. (Shiga, Japan). The forward and reverse primers for CAT, CuZn-SOD, Mn-SOD, GPx, and GAPDH (Table 1) were synthesized by Bio Basic, Inc. (Markham, Ontario, Canada). All other laboratory chemicals were of the highest available purity from commercial suppliers.

2.2. Animal Treatments. Seven-week-old male ICR mice were obtained from the National Laboratory Animal Center (Mahidol University, Thailand). All mice were housed on wood chip bedding in polysulfone cages with water and commercial regular diet (RD, SmartHeart ${ }^{\oplus}$ from Perfect Companion Pet Care Company, Thailand; $24 \%$ crude protein, $4.5 \%$ crude fat, $5 \%$ fiber, $10 \%$ moisture, $10 \%$ ash, $1 \%$ calcium,
0.7\% phosphorous, and vitamins $\mathrm{A}, \mathrm{D} 3$, and E) supplied ad libitum in the Northeast Laboratory Animal Center, Khon Kaen University, Thailand, and acclimated for a week before dosing under the supervision of the Animal Ethics Committee for Use and Care of Khon Kaen University (approval number AEKKU 92/2555). The mice quarters were air conditioned $\left(23 \pm 2^{\circ} \mathrm{C}\right)$ and controlled for humidity $45 \pm 2 \%$ $\mathrm{RH}$ and had a $12 \mathrm{~h}$ light/dark cycle.

The high-fat, high-fructose diet (HFFD) mice were additionally given hydrogenated soybean oil intragastrically $(1 \mathrm{~mL} /$ day), which consisted of $44.1 \%(\mathrm{w} / \mathrm{w})$ saturated fat and $0.2 \%(\mathrm{w} / \mathrm{w})$ trans fatty acid (certified by Institute of Nutrition, Mahidol University, Thailand), with the addition of $20 \%(\mathrm{w} / \mathrm{v})$ fructose in their drinking water supplied ad libitum for 2, 4, and 8 weeks ( $n=5$ for each group). The control mice $(n=5)$ were fed with commercial regular diet (RD) and water ad libitum for 4 weeks. The ages of all of the mice at the end of treatment were between 10 and 16 weeks, consistent with adult-aged mice (2-4 months). Levels of antioxidant enzymes and lipid peroxidation, including the GSH/GSSG ratio, have been proposed to be equivalent in the same age-range, for example, young (4-6 weeks), adult (3 months), middle-aged (9 months), and aged (18-24 months) [14]. All mice were sacrificed $24 \mathrm{~h}$ after the last treatment, and their organs (livers and brains) were immediately excised and either used for the isolation of total RNA or immediately stored at $-80^{\circ} \mathrm{C}$ for further analysis.

2.3. Tissue Fixation, Processing, and Staining. The liver tissue was prepared for histomorphological examination according to the method of Jearapong et al. [15] with some modifications. Briefly, a fragment of liver tissue was washed immediately in phosphate buffered saline then fixed by immersion in $10 \%$ neutral-buffered-formalin overnight and dehydrated for paraffin embedding. The paraffin piece was cut into $5 \mathrm{~mm}$ sections using a microtome (Microm HM315, Thermo Scientific, Walldorf, Germany) and placed on a microscope slide. The embedded tissue was stained with hematoxylin and eosin (H\&E) and evaluated for histomorphological features at 200x magnification using an Axiostar Plus microscope (Carl Ziess, Oberkochen, Germany).

2.4. Semiquantification of CAT, SOD, and GPX mRNA Levels. Mouse CAT, CuZn-SOD, Mn-SOD, GPx, and glyceraldehyde 3-phosphate dehydrogenase (GAPDH) mRNAs were semiquantified using RT-PCR. Total RNA was reverse-transcribed using ReverTraAce (Toyobo Co., Ltd.) [16] and the cDNA was amplified with specific primers under the conditions recommended by the supplier, Vivantis Technologies Sdn. 
TABLE 2: PCR programs for mouse CAT, CuZn-SOD, Mn-SOD, GPx, and GAPDH.

\begin{tabular}{lcccccc}
\hline Gene & Denaturation $\left({ }^{\circ} \mathrm{C} / \mathrm{sec}\right)$ & Annealing $\left({ }^{\circ} \mathrm{C} / \mathrm{sec}\right)$ & Extension $\left({ }^{\circ} \mathrm{C} / \mathrm{sec}\right)$ & Number of cycles & Product size (bp) & References \\
\hline CAT & $94 / 45$ & $55 / 60$ & $72 / 60$ & 31 & 229 & {$[17]$} \\
CuZn-SOD & $94 / 45$ & $56 / 60$ & $72 / 60$ & 26 & 246 & {$[18]$} \\
Mn-SOD & $94 / 45$ & $55 / 60$ & $72 / 60$ & 35 & 241 & {$[18]$} \\
GPx & $94 / 45$ & $56 / 60$ & $72 / 60$ & 28 & 197 & {$[18]$} \\
GAPDH & $95 / 30$ & $64 / 30$ & $72 / 60$ & 28 & 145 & {$[19]$} \\
\hline
\end{tabular}

Bhd. (Selangor Darul Ehsan, Malaysia). The PCR program conditions were set according to the methods of Chatuphonprasert et al. [17], Lao-Ong et al. [18], and Kondo et al. [19] with some modifications (Table 2). The PCR products were separated using $1.5 \%$ agarose gel electrophoresis and detected under ultraviolet (UV) light in the presence of Novel Juice from GeneDirex ${ }^{\bullet}$ (Bio-Helix Co., Ltd., Taiwan). The target PCR products were normalized to GAPDH and semiquantified using a Syngene ${ }^{\oplus}$ gel documentation machine (Ingenius L, Cambridge, UK) and the GeneTools match program (Syngene).

2.5. Determination of SOD Activity. The level of SOD activity in liver and brain homogenates was determined by the inhibition of formazan formation [17]. A chloroform and ethanol extract of liver or brain homogenate was mixed with the reagent mixture of xanthine, ethylenediamine tetraacetic acid (EDTA), NBT, $\mathrm{Na}_{2} \mathrm{CO}_{3}$, and BSA, followed by xanthine oxidase. The reaction was incubated at $25^{\circ} \mathrm{C}$ for $20 \mathrm{~min}$ and terminated with $\mathrm{CuCl}_{2}$. The absorbance of formazan was measured at a wavelength of $550 \mathrm{~nm}$. The percentage of formazan inhibition was compared with a bovine CuZn-SOD standard.

2.6. Determination of CAT Activity. The level of CAT activity was assessed according to the method of Chatuphonprasert et al. [17]. Briefly, sample homogenates were incubated in a $\mathrm{H}_{2} \mathrm{O}_{2}$ substrate at $37^{\circ} \mathrm{C}$ for $1 \mathrm{~min}$ before the addition of ammonium molybdate. The absorbance of the resulting yellow complex was measured at a wavelength of $405 \mathrm{~nm}$. CAT activity is measured as the percentage inhibition of complex formation compared with a bovine hepatic CAT standard.

2.7. Determination of GPx Activity. The level of GPx activity was determined according to the method of Chatuphonprasert et al. [17]. The reaction mixture, consisting of sample homogenate, sodium phosphate buffer ( $\mathrm{pH}$ 7.4), EDTA, and sodium azide, was incubated at room temperature for $10 \mathrm{~min}$ followed by the addition of the GSH substrate. The reaction was initiated by adding $\mathrm{H}_{2} \mathrm{O}_{2}$ and terminated using 5sulfosalicylic acid (SSA). The reaction mixture was then centrifuged at 1,900 rpm for $15 \mathrm{~min}$, and the GSSG content of the supernatant was used to assess GPx activity. The results are expressed as unit/mg protein, where a unit of GPx activity was defined as mmol of GSSG formed $/ \mathrm{min}$ at $30^{\circ} \mathrm{C}$ and $\mathrm{pH} 7.4$ [19].
2.8. Assessment of the Total Glutathione Content and the GSH to GSSG Ratio. Glutathione content was determined according to the method of Chatuphonprasert et al. [17]. The sample homogenate was deproteinized with SSA and kept at $2-8^{\circ} \mathrm{C}$ for $10 \mathrm{~min}$ before being centrifuged at $10,000 \times \mathrm{g}$ at $4^{\circ} \mathrm{C}$ for $10 \mathrm{~min}$. For the determination of total glutathione, the supernatant was mixed with the reaction mixture, which consisted of EDTA, NADPH, DTNB, and glutathione reductase in PBS buffer ( $\mathrm{pH}$ 7.0). The absorbance of the thiol anions at a wavelength of $405 \mathrm{~nm}$ was measured every $60 \mathrm{~s}$ for $5 \mathrm{~min}$ using a spectrophotometer. To determine the GSSG content, the homogenate was treated with 4-VP before the addition of the reaction mixture and then incubated at room temperature for $1 \mathrm{~h}$. Total glutathione, GSH, and GSSG contents were determined by comparing the net slope of the samples with the slope of the standard curve of GSH or GSSG [17].

2.9. Determination of Lipid Peroxidation. The thiobarbituric acid (TBA) assay was used to determine lipid peroxidation according to the method of Chatuphonprasert et al. [17] with a few modifications. The sample homogenates or MDA standards were incubated at $37^{\circ} \mathrm{C}$ for $1 \mathrm{~h}$ before addition of trichloroacetic acid, TBA, and acetic acid. The reaction mixtures were then boiled for $15 \mathrm{~min}$, and the TBA-reactive species (TBARS) were quantified using a spectrofluorometer at an emission wavelength of $551 \mathrm{~nm}$ and an excitation wavelength of $528 \mathrm{~nm}$.

2.10. Statistical Analysis. The results were analyzed using a one-way analysis of variance (ANOVA) followed by Tukey's post hoc test (version 17; SPSS Inc., Chicago, IL, USA). $p<$ 0.05 was considered to be statistically significant.

\section{Results}

3.1. Histomorphological Features of the Livers of Mice Fed a High-Fat, High-Fructose Diet. Micrographs of H\&E stained mouse hepatic tissues are shown in Figure 1. Compared with the mice fed the regular diet (Figure 1(a)), the liver sections of the mice fed the HFFD for 2 weeks exhibited steatosis as hepatocytes containing microvesicular fat droplets as clear areas (Figure 1(b)). After 4 weeks of the HFFD, the liver sections showed additional fat droplet accumulation and hepatocyte degeneration as sinusoidal pathology (Figure 1(c)). These pathological changes increased after 8 weeks of HFFD treatment with both microvesicular and macrovesicular fat 


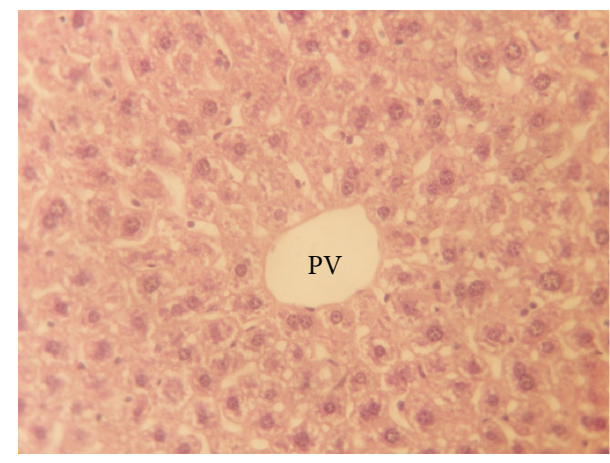

(a) Regular diet

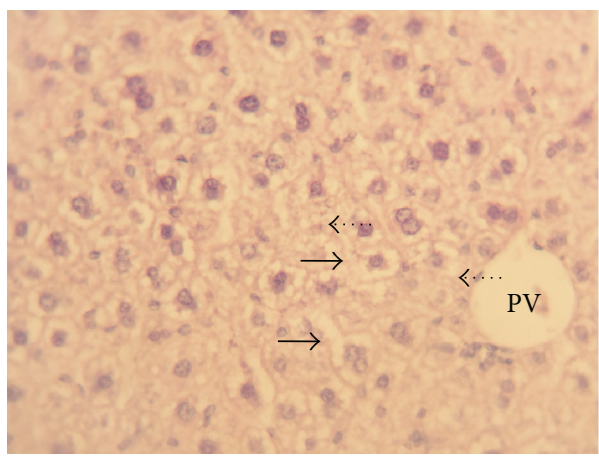

(c) High-fat-high-fructose diet, 4 weeks

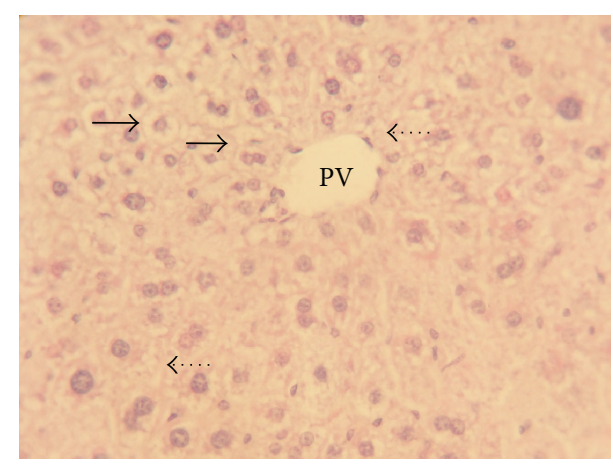

(b) High-fat-high-fructose diet, 2 weeks

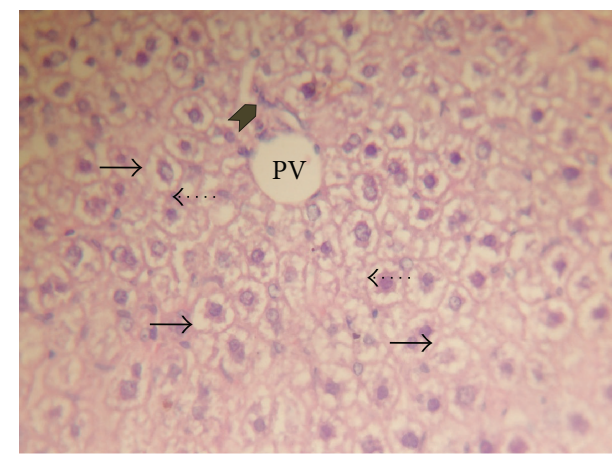

(d) High-fat-high-fructose diet, 8 weeks

FIGURE 1: Histopathology of hematoxylin and eosin (H\&E) stained mouse livers. The livers were collected 24 hours after the last treatment. Mice were fed a regular diet (a), or a high-fat, high-fructose diet for 2 weeks (b), 4 weeks (c), or 8 weeks (d). The micrographs are shown at a magnification of 400 -fold. The solid arrows indicate sinusoidal pathology, the dash arrows indicate fat droplets, and the gray arrow indicates the foci of lobular inflammation in the liver. PV: portal vein.

droplets observed throughout the liver sections along with increased sinusoidal pathology and foci of inflammation (Figure 1(d)).

\subsection{Antioxidant System Profile in the Livers of Mice Fed a High-} Fat, High-Fructose Diet. The level of CuZn-SOD mRNA was significantly upregulated in the livers of mice fed the HFFD (Figure 2). The mRNA level of CuZn-SOD continually increased, with the highest expression after 4 weeks followed by a gradual decline at 8 weeks (Figure 2). The level of $\mathrm{Mn}$ SOD mRNA was significantly increased in the HFFD-fed mouse livers. The level of CAT mRNA was similar to that of CuZn-SOD, while the level of GPx mRNA significantly increased for the first two weeks of the HFFD treatment but then started to decline at 4 weeks to nearly the same level as that of the normal mice at 8 weeks. Corresponding to the levels of mRNA expression (Figure 2), SOD, CAT, and GPx activities were significantly elevated in the mouse livers throughout the HFFD treatment for 8 weeks (Figures 3(a)$3(\mathrm{c})$ ), though the enzymatic activities notably declined at 8 weeks. The total glutathione content and the GSH/GSSG ratio decreased sharply at all stages of the HFFD treatment whereas there was a significant increase in GSSG (Figure 4), indicating the depletion of the GSH stores. Moreover, the level of lipid peroxidation, presented as the concentration of MDA, was excessively raised in the HFFD mouse livers throughout the treatment period (Figure 5).

3.3. Antioxidant System Profile in the Brains of Mice Fed a High-Fat, High-Fructose Diet. The levels of all investigated antioxidant mRNAs, including CuZn-SOD, Mn-SOD, CAT, and GPx, were significantly increased in the HFFD mouse brains, with the highest levels at week 4 , followed by a gradual decline at 8 weeks (Figure 6). The patterns of SOD, CAT, and GPx activities in the brains corresponded with the mRNA levels (Figure 7); the enzymatic activities gradually but significantly increased in the HFFD mouse brains and reached the maximal level at week 4 before dropping at week 8. The total glutathione content and the GSH/GSSG ratio were greatly reduced, with a significant increase in GSSG content throughout the duration of the HFFD treatment (Figure 8). Additionally, a significant increase in MDA production was observed in the HFFD mouse brains (Figure 9). These observations suggest that enhanced lipid peroxidation occurred in the HFFD mouse brains.

\section{Discussion}

Excess consumption of carbohydrates (fructose and sucrose) and fats (fatty acids and cholesterol) plays a key role in 


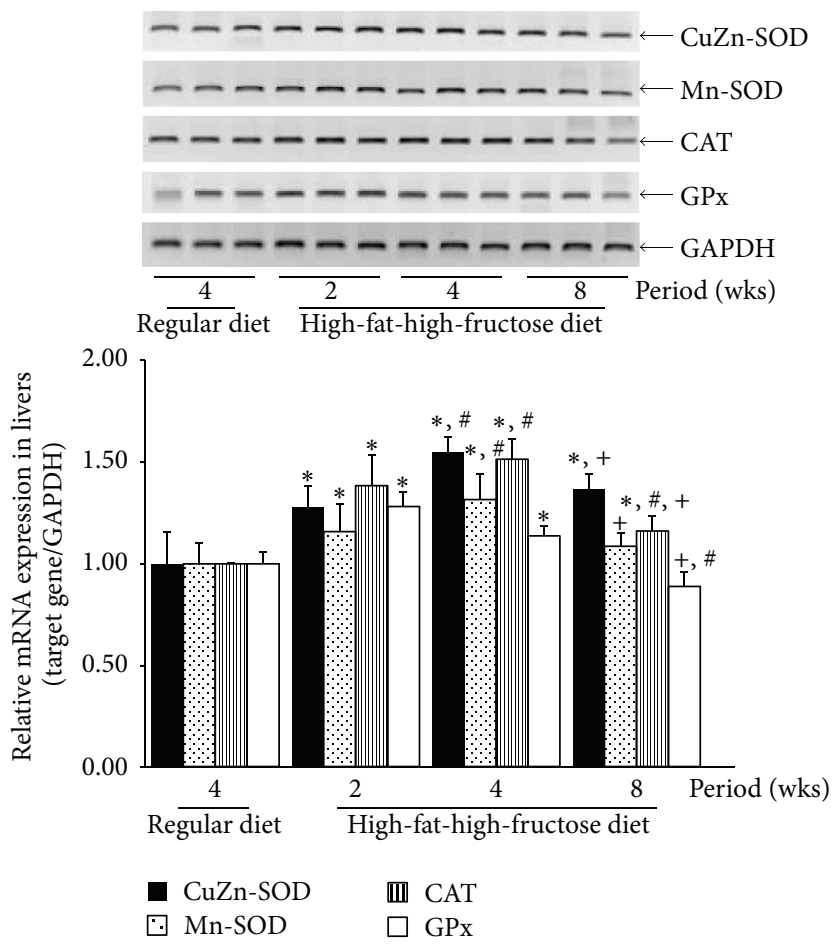

Figure 2: Effects of a high-fat, high-fructose diet on CuZn-SOD, Mn-SOD, CAT, and GPx mRNA expression in mouse livers. Mice were fed a commercial standard diet for 4 weeks, and a high-fat, high-fructose diet (HFFD) was administered intragastrically for 2, 4, or 8 weeks. A semiquantitative determination of the hepatic mRNA expression of certain genes was performed using pairs of primers specific to the investigated genes. The data are presented as the mean \pm SD $(n=5)$. Significant differences were determined using a one-way analysis of variance (ANOVA) followed by Tukey's post hoc test. ${ }^{*} p<0.05$ versus the regular diet mice; ${ }^{*} p<0.05$ versus 2 -week HFFD mice; ${ }^{+} p<0.05$ versus 4 -week HFFD mice.

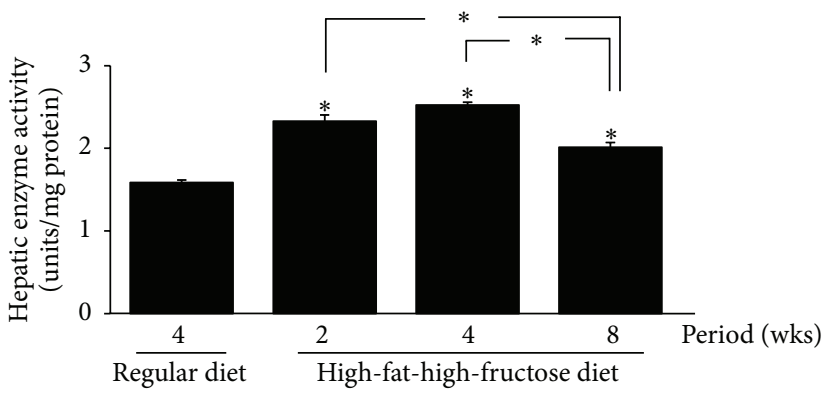

(a) SOD

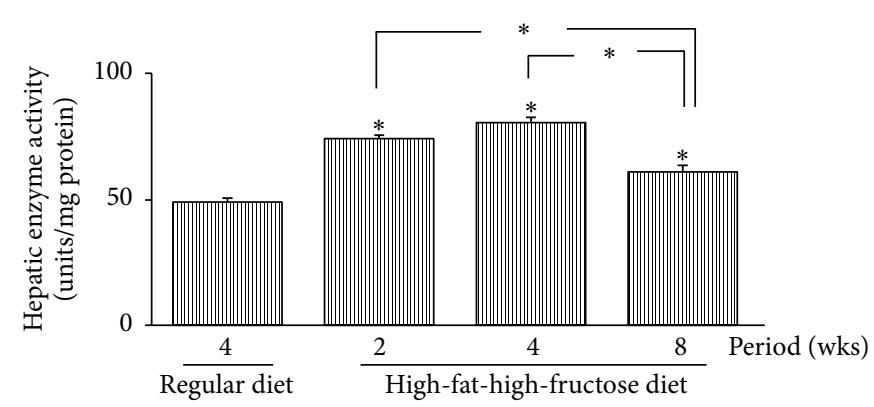

(b) CAT

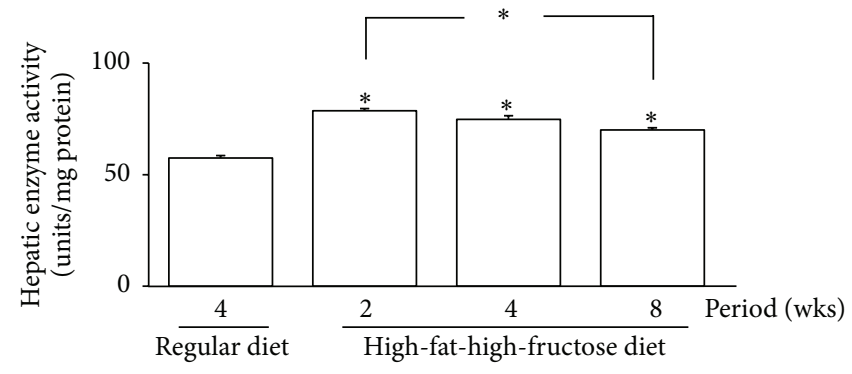

(c) GPx

FIGURE 3: Effects of a high-fat, high-fructose diet on hepatic SOD, CAT, and GPx enzymatic activity. Mice were fed a commercial standard diet for 4 weeks, and a high-fat, high-fructose diet (HFFD) was administered intragastrically for 2, 4, or 8 weeks. The mice were sacrificed $24 \mathrm{~h}$ after the last treatment, and their livers were immediately excised to determine the activities of the SOD, CAT, and GPx enzymes. The data are presented as the mean $\pm \mathrm{SD}(n=5)$. Significant differences were determined using a one-way analysis of variance (ANOVA) followed by Tukey's post hoc test. ${ }^{*} p<0.05$ versus the regular diet mice. 


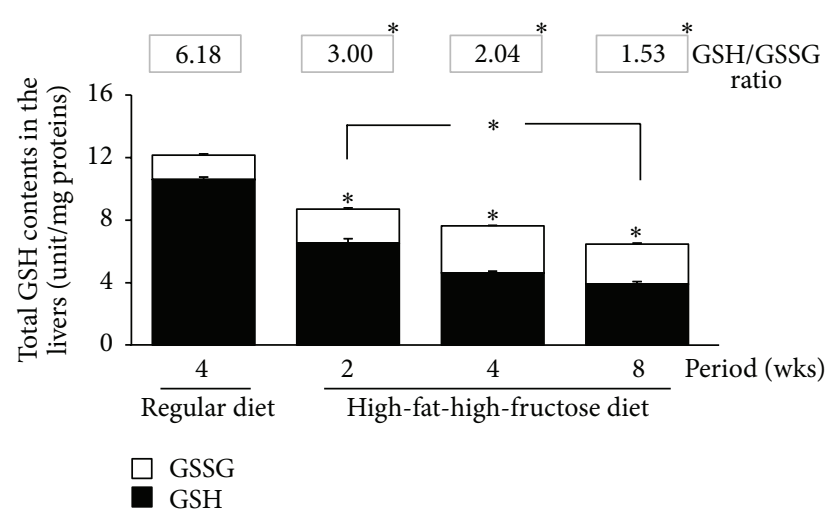

FIGURE 4: Effects of a high-fat, high-fructose diet on the total GSH, reduced GSH, and GSSH content and the GSH/GSSG ratio in the mouse livers. Mice were fed a commercial standard diet for 4 weeks, and a high-fat, high-fructose diet (HFFD) was administered intragastrically for 2 , 4 , or 8 weeks. The mice were sacrificed $24 \mathrm{~h}$ after the last treatment, and their livers were immediately excised to examine the total glutathione, reduced GSH (black column), and GSSG (white column) contents, and the GSH/GSSH ratio was calculated (digit above the graph). The data are presented as the mean $\pm \mathrm{SD}(n=5)$. Significant differences were determined using a one-way analysis of variance (ANOVA) followed by Tukey's post hoc test. ${ }^{*} p<0.05$ versus the regular diet mice.

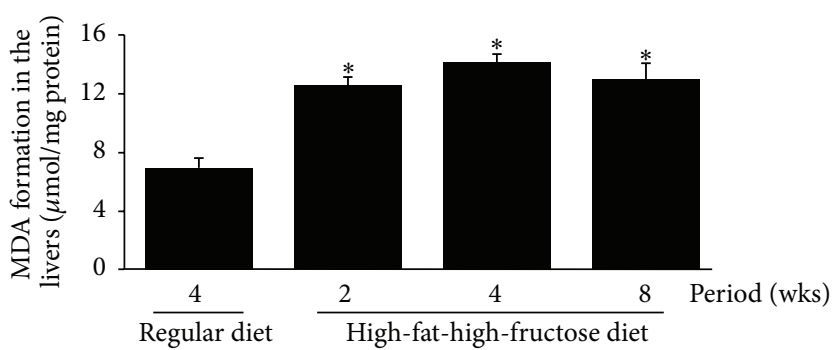

FiguRE 5: Effects of a high-fat, high-fructose diet on lipid peroxidation in the mouse livers. Mice were fed a commercial standard diet for 4 weeks, and a high-fat, high-fructose diet (HFFD) was administered intragastrically for 2, 4, or 8 weeks. The mice were sacrificed $24 \mathrm{~h}$ after the last treatment, and their livers were immediately excised to determine the levels of malondialdehyde (MDA). The data are presented as the mean \pm SD $(n=5)$. Significant differences were determined using a one-way analysis of variance (ANOVA) followed by Tukey's post hoc test. ${ }^{*} p<0.05$ versus the regular diet mice.

the development and progression of NAFLD through the activation of high-fat diet-modulated lipid metabolic pathways $[13,20]$. Excessive carbohydrate and/or fat intake has also been shown to increase the concentrations of both blood glucose and free fatty acids, contributing to the accumulation of neutral lipids in the liver [21]. The hepatic metabolites of fructose, namely, fructose-1-phosphate and fructose-1,6-bisphosphate, further increase the storage of free fatty acids (FFA) in the liver and can cause significantly higher adenosine triphosphate (ATP) degradation and uric acid production in cirrhotic patients [22]. The lipogenesis of FFA in the liver subsequently causes impaired glycogen synthesis and insulin resistance in hepatocytes [23, 24] and adipocytokine release causes hepatocyte lipotoxicity, which damages hepatocytes through induction of apoptosis and recruitment of proinflammatory mediators [25]. In the present study, we fed mice a high-fat, high-fructose diet (HFFD) which has been shown to cause overnutrition in ICR mice $[26,27]$. The energy from fat and fructose in the HFFD was $49.6 \%$ and $28.0 \%$, of the total energy given and additional energy intake of $9.2 \mathrm{kcal} /$ day. Lieber et al. [28] reported a dietary model of NASH that used a high fat diet
( $71 \%$ of energy from fat) in rats. Rats fed the high-fat diet for 3 weeks showed insulin resistance, marked panlobular steatosis, and hepatic lipid accumulation. In addition, hepatic oxidative damage, steatosis, and inflammation were noted in the rats fed high-fat diet, and these abnormal symptoms were reduced using a restricted dietary consumption program [29]. This corresponds to our results in mice fed the HFFD. Microvesicular fat droplets were observed after 2 weeks of HFFD treatment, and inflammatory histopathology was found in the mouse livers at 8 weeks.

The progression of nonalcoholic steatosis to steatohepatitis has been linked to the action of reactive oxidant species (ROS) in the liver [30, 31]. ROS are generated in the liver through several pathways, such as the mitochondria, peroxisomes, cytochrome P-450, reduced nicotinamide adenine dinucleotide oxidase, cyclooxygenase, and lipoxygenase. In both NASH and experimental steatohepatitis, the expression of hepatic CYP2E1 increased, leading to oxidative stress [32]. Oxidative stress can damage proteins or unsaturated lipids in cell membrane [33]. Here we report that MDA levels were elevated in the liver and brain of mice fed the HFFD, indicating that lipid peroxidation increased in the 


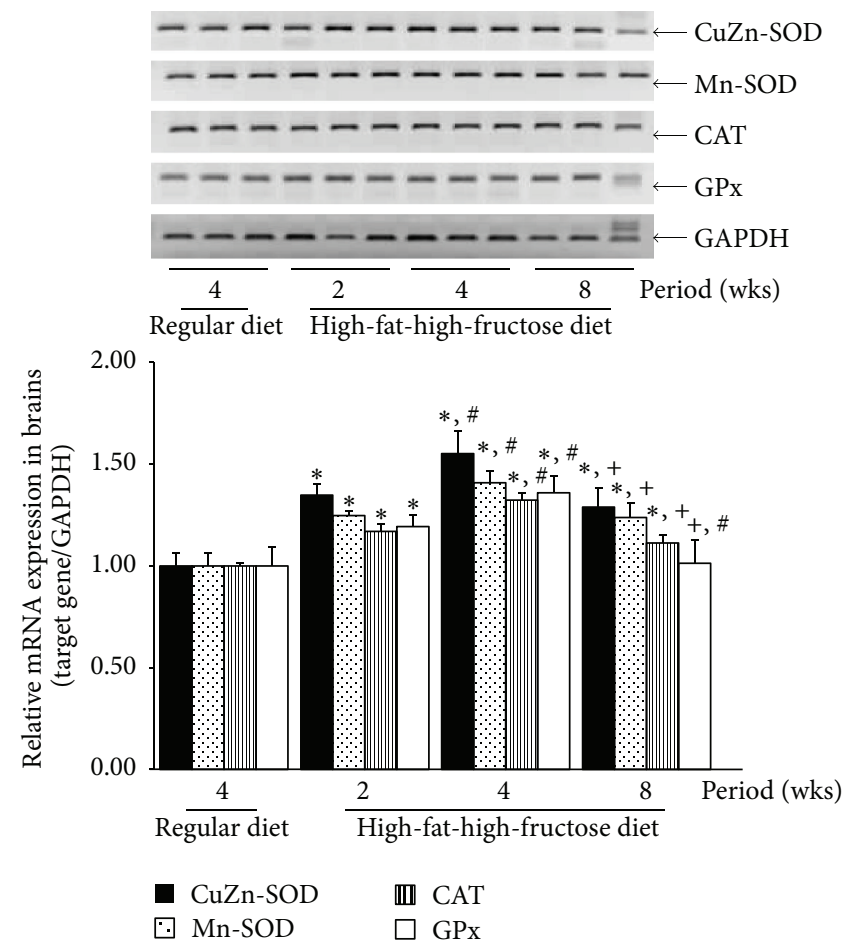

FIGURE 6: Effects of a high-fat, high-fructose diet on CuZn-SOD, Mn-SOD, CAT, and GPx mRNA expression in the mouse brains. Mice were fed a commercial standard diet for 4 weeks, and a high-fat, high-fructose diet (HFFD) was administered intragastrically for 2, 4, or 8 weeks. A semiquantitative determination of the mRNA expression of certain genes in mouse brain was performed using pairs of primers specific to the investigated genes. The data are presented as the mean $\pm \operatorname{SD}(n=5)$. Significant differences were determined using a one-way analysis of variance (ANOVA) followed by Tukey's post hoc test. ${ }^{*} p<0.05$ versus the regular diet mice; ${ }^{\#} p<0.05$ versus 2 -week HFFD mice; ${ }^{+} p<0.05$ versus 4 -week HFFD mice.

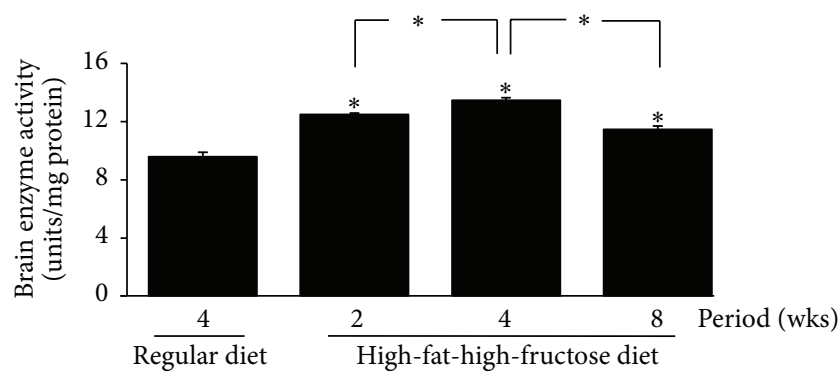

(a) SOD

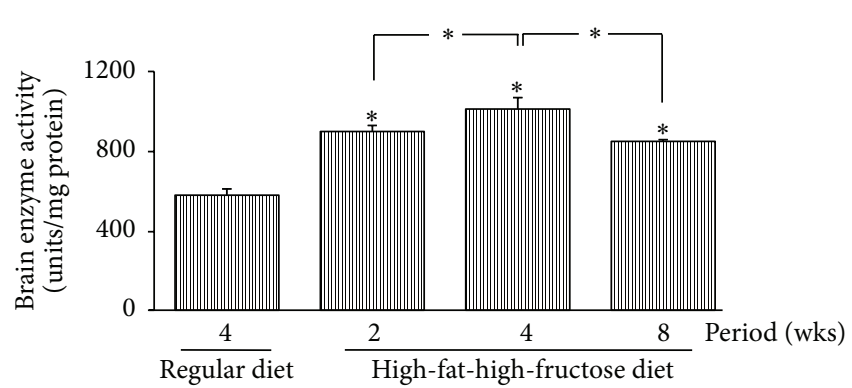

(b) CAT

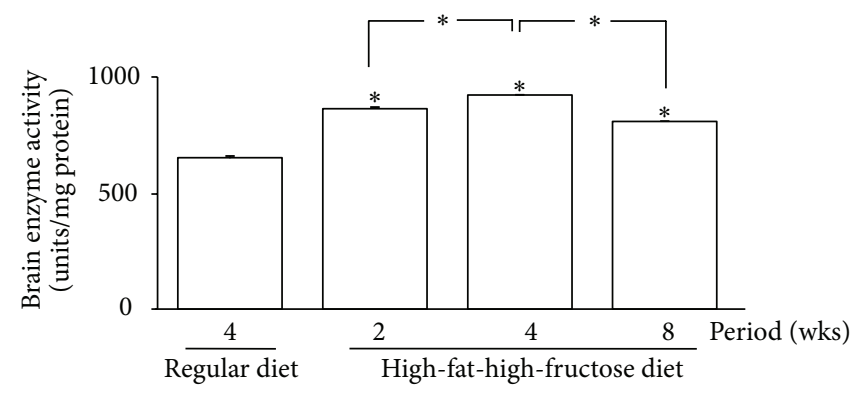

(c) GPx

FIGURE 7: Effects of a high-fat, high-fructose diet on the SOD, CAT, and GPx enzymatic activity in the mouse brains. Mice were fed a commercial standard diet for 4 weeks, and a high-fat, high-fructose diet (HFFD) was administered intragastrically for 2, 4, or 8 weeks. The mice were sacrificed $24 \mathrm{~h}$ after the last treatment, and their brains were immediately excised to determine the SOD, CAT, and GPx enzyme activities. The data are presented as the mean \pm SD $(n=5)$. Significant differences were determined using a one-way analysis of variance (ANOVA) followed by Tukey's post hoc test. ${ }^{*} p<0.05$ versus the regular diet mice. 


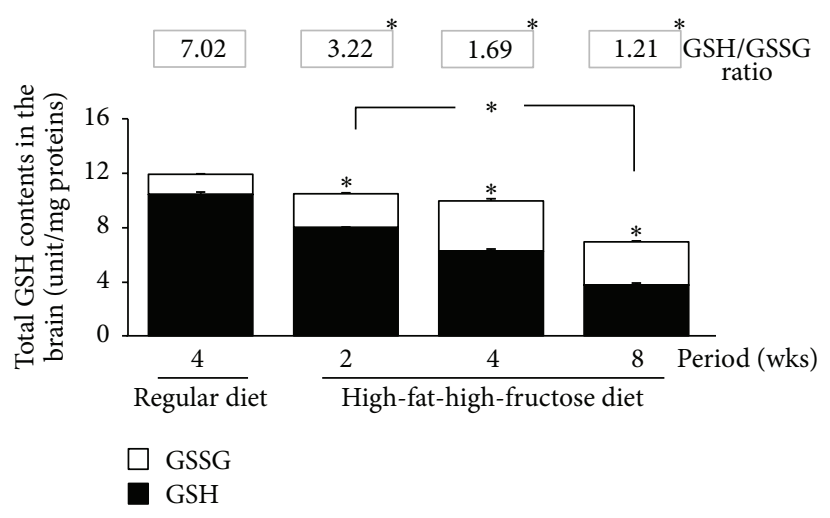

FIGURE 8: Effects of a high-fat, high-fructose diet on the total GSH, reduced GSH, and GSSH content and the GSH/GSSG ratio in the mouse brains. Mice were fed a commercial standard diet for 4 weeks, and a high-fat, high-fructose diet (HFFD) was administered intragastrically for 2,4 , or 8 weeks. The mice were sacrificed $24 \mathrm{~h}$ after the last treatment; their brains were immediately excised to determine the total glutathione, reduced GSH (black column), and GSSG (white column) content, and the GSH/GSSH ratio was then calculated (digit above the graph). The data are presented as the mean \pm SD $(n=5)$. Significant differences were determined using a one-way analysis of variance (ANOVA) followed by Tukey's post hoc test. ${ }^{*} p<0.05$ versus the regular diet mice.

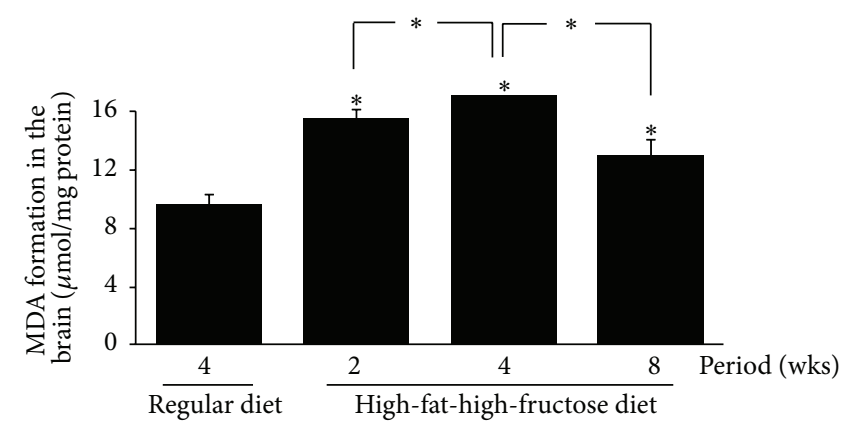

FiguRE 9: Effects of a high-fat, high-fructose diet on lipid peroxidation in the mouse brains. Mice were fed a commercial standard diet for 4 weeks, and a high-fat, high-fructose diet (HFFD) was administered intragastrically for 2,4 , or 8 weeks. The mice were sacrificed $24 \mathrm{~h}$ after the last treatment, and their brains were immediately excised to determine the levels of malondialdehyde (MDA). The data are presented as the mean \pm SD $(n=5)$. Significant differences were determined using a one-way analysis of variance (ANOVA) followed by Tukey's post hoc test. ${ }^{*} p<0.05$ versus the regular diet mice.

HFFD-fed mice presumably due to oxidative stress. We then examined the antioxidant systems through SOD, CAT, and GPx mRNA expression levels and enzymatic activity, together with the nonenzymatic antioxidant glutathione system [34, 35]. Dhuley reported the elevation of SOD, CAT, and GPx, in the livers and hearts of rats receiving a diet supplemented with $20 \%$ coconut oil for 90 days as well as increased MDA and hydroperoxide levels along with a decrease in glutathione content [36]. A study comparing oxidative stress in chronic hepatitis patients with NAFLD patients showed that the MDA level and the GPx and SOD activities were elevated, while the CAT activity and the glutathione content were suppressed in the plasma of the NAFLD patients [34]. Here, we present evidence that the mRNA expression and enzymatic activity of SOD, CAT, and GPx were increased and the total glutathione, the reduced GSH, and the GSH/GSSG ratio were decreased in both livers and brains of mice fed the HFFD for 2-8 weeks.

The cytosolic (CuZn-SOD) and mitochondrial (MnSOD) SOD enzymes catalyze the dismutation of superoxide $\left(\mathrm{O}_{2}{ }^{-}\right)$into oxygen $\left(\mathrm{O}_{2}\right)$ and hydrogen peroxide $\left(\mathrm{H}_{2} \mathrm{O}_{2}\right)$ [37].
Together, the SOD enzymes keep the intracellular steadystate concentration of $\left(\mathrm{O}_{2}{ }^{-}\right)$low, while CAT and GPx remove the $\mathrm{H}_{2} \mathrm{O}_{2}$ generated from the SOD reaction. GPx uses GSH to catalyze the transformation of $\mathrm{H}_{2} \mathrm{O}_{2}$ (or $\mathrm{ROOH}$ ) to $\mathrm{H}_{2} \mathrm{O}$ (or ROH) and GSSG [38]. In the livers and brains of the HFFD-fed mice in the present study, GPx activity and GSSG content increased, while GSH content decreased, reducing the GSH/GSSG ratio. These findings indicate that the HFFD caused oxidative stress due to increased production of superoxide (SOD) and hydrogen peroxide (CAT and GPx).

\section{Conclusion}

The continuous consumption of a high-fat, high-fructose diet caused oxidative stress in mice: SOD, CAT, and GPx enzyme activities increased, while GSH stores were depleted. This manifested as histopathological damage to the liver including steatosis, inflammation, and damage to hepatocytes. Thus, the oxidant-antioxidant imbalance induced by long-term consumption of a high-fat, high-fructose diet appears to 
contribute to the development of NAFLD and increase the risk of progression to NASH.

$\begin{array}{ll}\text { Abbreviations } \\ \text { CAT: } & \text { Catalase } \\ \text { CuZn-SOD: } & \text { Copper-zinc superoxide dismutase } \\ \text { GPx: } & \text { Glutathione peroxidase } \\ \text { GSH: } & \text { Glutathione reduced form } \\ \text { GSSG: } & \text { Glutathione oxidized form } \\ \text { HFFD: } & \text { High-fat, high-fructose diet } \\ \text { Mn-SOD: } & \text { Manganese superoxide dismutase } \\ \text { NAFLD: } & \text { Nonalcoholic fatty liver disease } \\ \text { NASH: } & \text { Nonalcoholic steatohepatitis } \\ \text { ROS: } & \text { Reactive oxygen species } \\ \text { SOD: } & \text { Superoxide dismutase. }\end{array}$

\section{Conflict of Interests}

There is no conflict of interests.

\section{Acknowledgments}

The Research Group for Pharmaceutical Activities of Natural Products Using Pharmaceutical Biotechnology (PANPB), Faculty of Pharmaceutical Sciences, and the Northeast Laboratory Animal Center, Khon Kaen University, Thailand, and the Faculty of Medicine, Mahasarakham University, Thailand, are acknowledged for providing research grants and facilities. Nattharat Jearapong expresses sincere gratitude for the Royal Golden Jubilee Ph.D. program scholarship (PHD/0197/ 2553). The authors wish to thank Tida Srikul, Mahasarakham University, for technical assistance and Dr. Glenn Borlace, Khon Kaen University, for English editing.

\section{References}

[1] M. Gaggini, M. Morelli, E. Buzzigoli, R. A. DeFronzo, E. Bugianesi, and A. Gastaldelli, "Non-alcoholic fatty liver disease (NAFLD) and its connection with insulin resistance, dyslipidemia, atherosclerosis and coronary heart disease," Nutrients, vol. 5, no. 5, pp. 1544-1560, 2013.

[2] C. Finelli and G. Tarantino, "Is visceral fat reduction necessary to favour metabolic changes in the liver?" Journal of Gastrointestinal and Liver Diseases, vol. 21, no. 2, pp. 205-208, 2012.

[3] G. Tarantino, C. Finelli, A. Colao et al., "Are hepatic steatosis and carotid intima media thickness associated in obese patients with normal or slightly elevated gamma-glutamyl-transferase?" Journal of Translational Medicine, vol. 10, article 50, 2012.

[4] J. D. Browning, L. S. Szczepaniak, R. Dobbins et al., "Prevalence of hepatic steatosis in an urban population in the United States: impact of ethnicity," Hepatology, vol. 40, no. 6, pp. 1387-1395, 2004.

[5] C. P. Day, "Pathogenesis of steatohepatitis," Best Practice \& Research Clinical Gastroenterology, vol. 16, pp. 663-678, 2002.

[6] S. Abiru, K. Migita, Y. Maeda et al., "Serum cytokine and soluble cytokine receptor levels in patients with non-alcoholic steatohepatitis," Liver International, vol. 26, no. 1, pp. 39-45, 2006.
[7] R. D. Situnayake, B. J. Crump, D. I. Thurnham, J. A. Davies, J. Gearty, and M. Davis, "Lipid peroxidation and hepatic antioxidants in alcoholic liver disease," Gut, vol. 31, no. 11, pp. 13111317, 1990.

[8] W. Wang, W. Zhou, B. Wang, H. Zhu, L. Ye, and M. Feng, "Antioxidant effect of apolipoprotein A-I on high-fat diet-induced non-alcoholic fatty liver disease in rabbits," Acta Biochimica et Biophysica Sinica, vol. 45, no. 2, pp. 95-103, 2013.

[9] F. A. Anania, "Non-alcoholic fatty liver disease and fructose: bad for us, better for mice," Journal of Hepatology, vol. 55, no. 1, pp. 218-220, 2011.

[10] M. B. Vos and J. E. Lavine, "Dietary fructose in nonalcoholic fatty liver disease," Hepatology, vol. 57, no. 6, pp. 2525-2531, 2013.

[11] Y. Yilmaz, "Review article: fructose in non-alcoholic fatty liver disease," Alimentary Pharmacology and Therapeutics, vol. 35, no. 10, pp. 1135-1144, 2012.

[12] M. Charlton, A. Krishnan, K. Viker et al., "Fast food diet mouse: novel small animal model of NASH with ballooning, progressive fibrosis, and high physiological fidelity to the human condition," The American Journal of Physiology-Gastrointestinal and Liver Physiology, vol. 301, pp. G825-G834, 2011.

[13] A. Ibrahim, S. Natarajan, and Ghafoorunissa, "Dietary transfatty acids alter adipocyte plasma membrane fatty acid composition and insulin sensitivity in rats," Metabolism: Clinical and Experimental, vol. 54, no. 2, pp. 240-246, 2005.

[14] S. L. van Dommelen, A. Rizzitelli, A. Chidgey, R. Boyd, K. Shortman, and L. Wu, "Regeneration of dendritic cells in aged mice," Cellular and Molecular Immunology, vol. 7, no. 2, pp. 108-115, 2010.

[15] N. Jearapong, W. Chatuphonprasert, and K. Jarukamjorn, "Effect of tetrahydrocurcumin on the profiles of drug-metabolizing enzymes induced by a high fat and high fructose diet in mice," Chemico-Biological Interactions, vol. 239, pp. 67-75, 2015.

[16] K. Jarukamjorn, T. Sakuma, J.-I. Miyaura, and N. Nemoto, "Different regulation of the expression of mouse hepatic cytochrome P450 2B enzymes by glucocorticoid and phenobarbital," Archives of Biochemistry and Biophysics, vol. 369, no. 1, pp. 89-99, 1999.

[17] W. Chatuphonprasert, L. Udomsuk, O. Monthakantirat, Y. Churikhit, W. Putalun, and K. Jarukamjorn, "Effects of Pueraria mirifica and miroestrol on the antioxidation-related enzymes in ovariectomized mice," Journal of Pharmacy and Pharmacology, vol. 65, no. 3, pp. 447-456, 2013.

[18] T. Lao-Ong, W. Chatuphonprasert, N. Nemoto, and K. Jarukamjorn, "Alteration of hepatic glutathione peroxidase and superoxide dismutase expression in streptozotocin-induced diabetic mice by berberine," Pharmaceutical Biology, vol. 50, no. 8, pp. 1007-1012, 2012.

[19] S. Kondo, W. Chatuphonprasert, A. Jaruchotikamol, T. Sakuma, and N. Nemoto, "Cellular glutathione content modulates the effect of andrographolide on $\beta$-naphthoflavone-induced CYP1A1 mRNA expression in mouse hepatocytes," Toxicology, vol. 280, no. 1-2, pp. 18-23, 2011.

[20] M. T. Timlin and E. J. Parks, "Temporal pattern of de novo lipogenesis in the postprandial state in healthy men," American Journal of Clinical Nutrition, vol. 81, no. 1, pp. 35-42, 2005.

[21] A. M. Zivkovic, J. B. German, and A. J. Sanyal, "Comparative review of diets for the metabolic syndrome: implications for nonalcoholic fatty liver disease," American Journal of Clinical Nutrition, vol. 86, no. 2, pp. 285-300, 2007.

[22] C. Loguercio, G. Nardone, P. Sicolo, R. Cuomo, C. Del Vecchio Blanco, and G. Budillon, "Intravenous load of fructose and 
fructose 1,6-diphosphate: effects on uricemia in patients with nonalcoholic liver disease," American Journal of Gastroenterology, vol. 91, no. 3, pp. 559-564, 1996.

[23] E. E. Kershaw and J. S. Flier, "Adipose tissue as an endocrine organ," Journal of Clinical Endocrinology and Metabolism, vol. 89, no. 6, pp. 2548-2556, 2004.

[24] T. Kadowaki, T. Yamauchi, N. Kubota, K. Hara, K. Ueki, and K. Tobe, "Adiponectin and adiponectin receptors in insulin resistance, diabetes, and the metabolic syndrome," The Journal of Clinical Investigation, vol. 116, no. 7, pp. 1784-1792, 2006.

[25] S. C. Cazanave, N. A. Elmi, Y. Akazawa, S. F. Bronk, J. L. Mott, and G. J. Gores, "CHOP and AP-1 cooperatively mediate PUMA expression during lipoapoptosis," American Journal of Physiology-Gastrointestinal and Liver Physiology, vol. 299, no. 1, pp. G236-G243, 2010.

[26] M. S. Winzell and B. Ahrén, "The high-fat diet-fed mouse: a model for studying mechanisms and treatment of impaired glucose tolerance and type 2 diabetes," Diabetes, vol. 53, supplement 3, pp. S215-S219, 2004.

[27] L. H. Tetri, M. Basaranoglu, E. M. Brunt, L. M. Yerian, and B. A. Neuschwander-Tetri, "Severe NAFLD with hepatic necroinflammatory changes in mice fed trans fats and a high-fructose corn syrup equivalent," American Journal of PhysiologyGastrointestinal and Liver Physiology, vol. 295, no. 5, pp. G987G995, 2008.

[28] C. S. Lieber, M. A. Leo, K. M. Mak et al., "Model of nonalcoholic steatohepatitis," The American Journal of Clinical Nutrition, vol. 79, no. 3, pp. 502-509, 2004.

[29] Y. Zou, J. Li, C. Lu et al., "High-fat emulsion-induced rat model of nonalcoholic steatohepatitis," Life Sciences, vol. 79, no. 11, pp. 1100-1107, 2006.

[30] C. P. Day and O. F. W. James, "Steatohepatitis: a tale of two 'Hits'?” Gastroenterology, vol. 114, no. 4, pp. 842-845, 1998.

[31] A. J. Sanyal, C. Campbell-Sargent, F. Mirshahi et al., "Nonalcoholic steatohepatitis: association of insulin resistance and mitochondrial abnormalities," Gastroenterology, vol. 120, no. 5, pp. 1183-1192, 2001.

[32] I. A. Leclercq, G. C. Farrell, J. Field, D. R. Bell, F. J. Gonzalez, and G. R. Robertson, "CYP2E1 and CYP4A as microsomal catalysts of lipid peroxides in murine nonalcoholic steatohepatitis," The Journal of Clinical Investigation, vol. 105, no. 8, pp. 1067-1075, 2000.

[33] J. W. Baynes, "Role of oxidative stress in development of complications in diabetes," Diabetes, vol. 40, no. 4, pp. 405-412, 1991.

[34] A. Kumar, A. Sharma, A. Duseja et al., "Patients with nonalcoholic fatty liver disease (NAFLD) have higher oxidative stress in comparison to chronic viral hepatitis," Journal of Clinical and Experimental Hepatology, vol. 3, no. 1, pp. 12-18, 2013.

[35] O. Zitka, S. Skalickova, J. Gumulec et al., "Redox status expressed as GSH:GSSG ratio as a marker for oxidative stress in paediatric tumour patients," Oncology Letters, vol. 4, no. 6, pp. 1247-1253, 2012.

[36] J. N. Dhuley, "Anti-oxidant effects of cinnamon (Cinnamomum verum) bark and greater cardamom (Amomum subulatum) seeds in rats fed high fat diet," Indian Journal of Experimental Biology, vol. 37, no. 3, pp. 238-242, 1999.

[37] R. Polavarapu, D. R. Spitz, J. E. Sim et al., "Increased lipid peroxidation and impaired antioxidant enzyme function is associated with pathological liver injury in experimental alcoholic liver disease in rats fed diets high in corn oil and fish oil," Hepatology, vol. 27, no. 5, pp. 1317-1323, 1998.
[38] B. Chance, H. Sies, and A. Boveris, "Hydroperoxide metabolism in mammalian organs," Physiological Reviews, vol. 59, no. 3, pp. 527-605, 1979. 

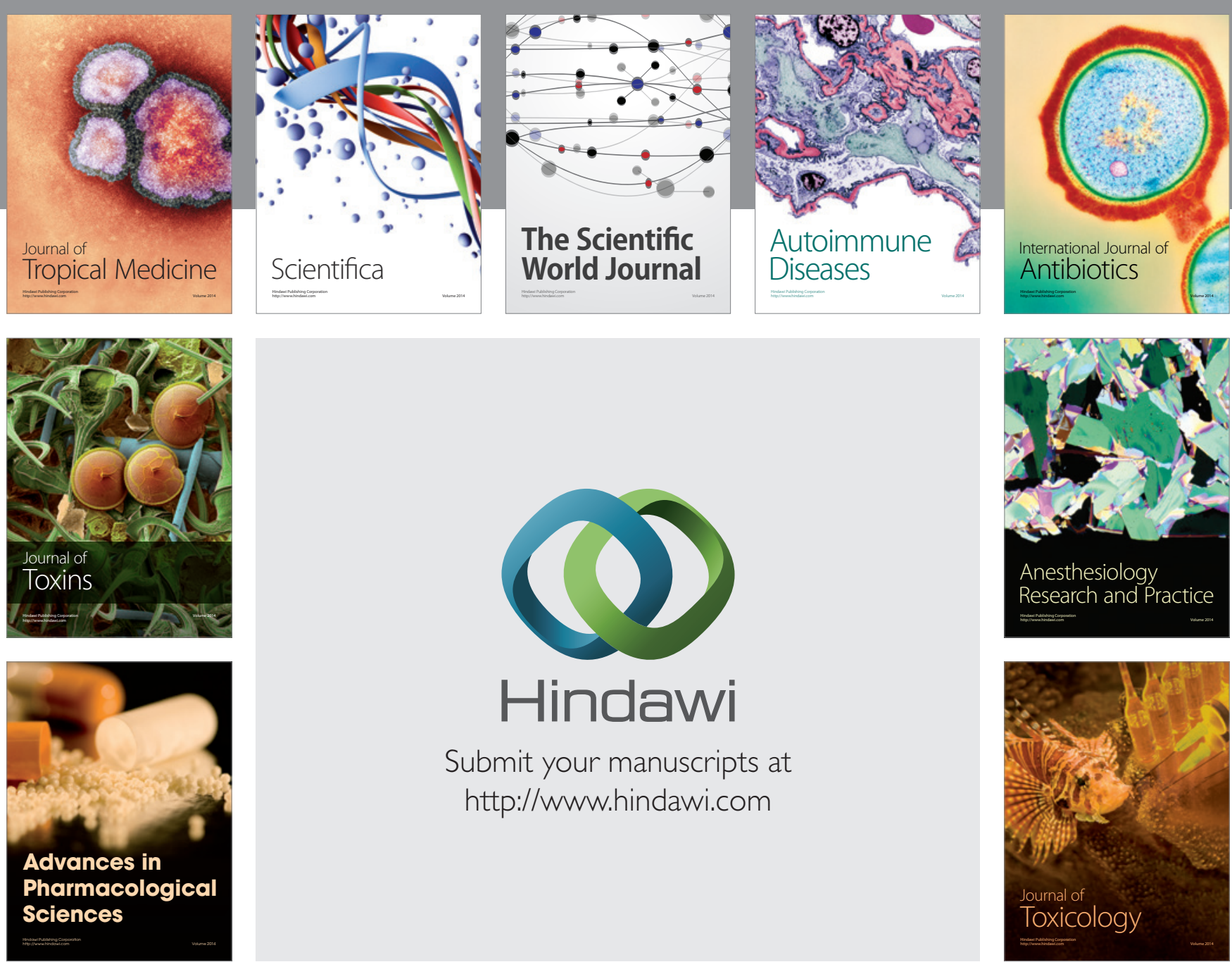

\section{Hindawi}

Submit your manuscripts at

http://www.hindawi.com
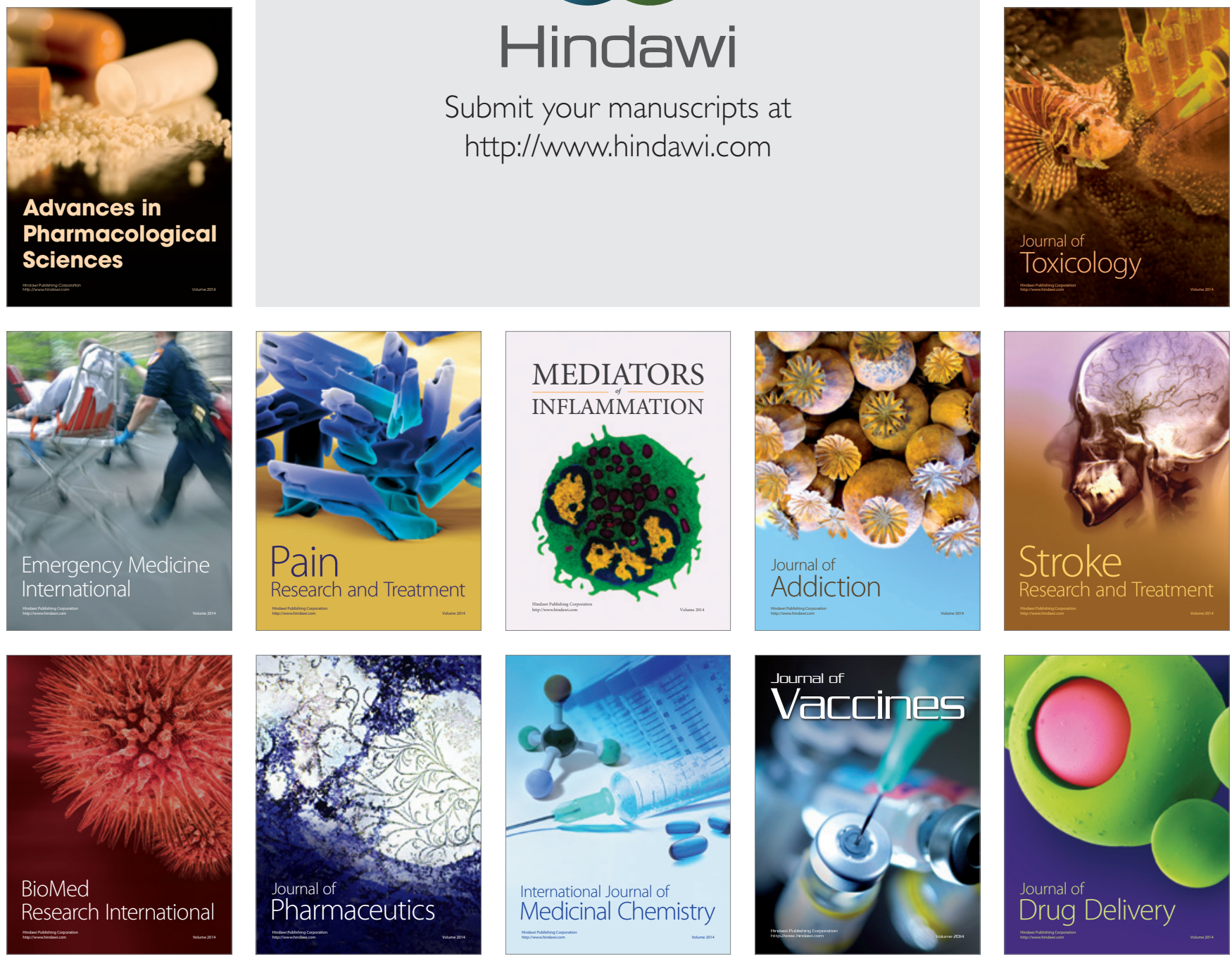